International Journal of Applied Mathematics

Volume 30 No. $6 \quad 2017,527-545$

ISSN: 1311-1728 (printed version); ISSN: 1314-8060 (on-line version)

doi: http://dx.doi.org/10.12732/ijam.v30i6.6

\title{
CONTROLLING THE FORCING OF THE LINEAR TRANSPORT EQUATION TO MEET AIR QUALITY NORMS AT EVERY POINT
}

\author{
D. Parra-Guevara ${ }^{1} \S$, Yu.N. Skiba ${ }^{2}$, D. Peña-Maciel ${ }^{3}$ \\ ${ }^{1,2,3}$ Center for Atmospheric Sciences \\ National Autonomous University of Mexico \\ Circuito Exterior, Ciudad Universitaria \\ CDMX, C. P. 04510, MEXICO
}

\begin{abstract}
A three-dimensional dispersion air pollution model for point, line or area sources is considered in a limited region. Particular solutions of such model and their respective maximum values are used to pose a quadratic programming problem with the aim to determine optimal emission rates of the sources and meet the standards of air quality at every point in a zone and each instant in an interval of time. The existence and uniqueness of the optimal control problem solution is proved. An efficient algorithm of successive orthogonal projections is used to calculate the optimal solution. Numerical examples obtained in the case of point sources demonstrate the method's ability.
\end{abstract}

AMS Subject Classification: 90C20, 49K20

Key Words: dispersion model, quadratic programming, optimal control

\section{Introduction}

The main objective of any air pollution control program is to establish a set of activities to reduce the concentration of each of atmospheric pollutants to air

Received: November 7, 2017

(c) 2017 Academic Publications

${ }^{\S}$ Correspondence author 
quality standards, or at least, minimize the number of hours or days when these standards are violated. All control programs can be classified in two categories: long-term and short-term strategies of control [5]. These programs complement each other, but differ in the methods and duration of their implementation. The long-term control (from several months to several years) is usually implemented for large-scale regions (from urban to global) in order to diminish emissions and reduce the mean concentration of the air pollutants. The replacement of fuel and introduction of new combustion technologies in factories and automobiles are typical actions that make up this control. Nevertheless, it is important to note that a long-term control cannot guarantee full protection for days with weak conditions for dispersion in the atmosphere, when the concentrations of air pollutants can reach dangerous levels. For such short events (from several hours to several days), a short-term control should be applied at the local (urban) scale, in order to ensure that the concentrations of pollutants meet air quality standards. Actions of this kind of control must provide immediate reduction in pollutant emissions and may even include stopping certain activities.

The idea of using mathematical programming models for environmental quality control comes from the late 60's, [2], [8]. In particular, the linear programming models proposed by Teller [22] and Kohn [10] for the abatement of pollutants in the atmosphere are the first optimal strategies that took into account economic and environmental variables. Since then, various air pollution control models have been posed for different purposes and polluted regions. The elements that characterize such optimization models are: i) the air pollutants under consideration, that is, primary or secondary substances which define the complexity of the chemical reactions to be considered in the dispersion model; ii) the objective function, or cost function that estimates the cost of changes in emission rates; iii) the environmental constraints to be fulfilled in a particular limited area (control zone); and iv) the technological constraints which define the upper and lower bounds for scarce resources or materials as fuels. Certainly, the different formulations of these elements produce linear or nonlinear programming models, which can be classified and solved as convex or nonconvex optimization problems. However, the fulfilment of ecological goals, technological restrictions and minimizing the costs associated with the reduction of emission rates are the common objectives of all air pollution control models [1], [4], [13], [14], [18].

In this work, it is assumed that a short-term forecast made with a dispersion model is adverse, i.e. the concentration of a pollutant emitted from several sources is above the respective air quality standard in a control zone. Then the problem of quadratic programming is formulated with the aim to calculate the 
damping coefficients and determine the optimum reduction in emission rates at the lowest price. We point out that the dispersion model solutions independently calculated for each source, along with theirs respective maximum values, play an important role in determining this control strategy. In our formulation of the problem, the quadratic objective function evaluates the cost of the control, while the linear constraints guarantee the fulfilment of the air quality norm at every point of the control zone and any moment of the control time interval. The existence and uniqueness of the optimal damping coefficients is proved. The quadratic programming problem is solved by the algorithm of successive orthogonal projections, which converges to the solution in a finite number of iterations. We emphasize that this control strategy is stricter than the strategies which are able to reduce the mean value concentrations of pollutants in the control zone or concentrations at specific control sites [13].

\section{Dispersion Model}

The dispersion of primary pollutants emitted into the atmosphere by point, line or area sources is usually described with a linear three-dimensional model. Dispersion models of such type are useful to establish the linear relationship between emissions and concentrations for single (passive) pollutants such as $\mathrm{CO}, \mathrm{SO}_{2}, \mathrm{NO}_{x}$ and soot [7]. In this section, a model of such type is formulated for one pollutant by means of the transport equation, and it is shown that this model is well-posed in the sense of Hadamard [9].

Let $\mathbf{D}=D \times(0, H)$ be a simply connected bounded domain in $\mathbf{R}^{3}$ with the boundary $\partial \mathbf{D}=S_{0} \cup S \cup S_{H}$ which is the union of the cylindric lateral surface $S$, the base $S_{0}$ at the bottom, and top cover $S_{H}$ at $z=H$ (see Fig. 1). The short-term dispersion model, considered for one passive pollutant in the domain $\mathbf{D}$ is defined by the following equations:

$$
\begin{gathered}
\frac{\partial \phi}{\partial t}+\mathbf{U} \cdot \nabla \phi+\sigma \phi-\nabla \cdot(\mu \nabla \phi)-\frac{\partial}{\partial z} \mu_{z} \frac{\partial \phi}{\partial z}+\nabla \cdot \phi^{s}=f(\mathbf{r}, t) \\
\phi^{s}=-\nu^{s} \phi \mathbf{e}_{3} \quad \text { in } \quad \mathbf{D} \\
\phi(\mathbf{r}, 0)=\phi^{0}(\mathbf{r}) \quad \text { in } \quad \mathbf{D} \\
\mu \nabla \phi \cdot \mathbf{n}-U_{n} \phi=0 \quad \text { on } \quad S^{-} \\
\mu \nabla \phi \cdot \mathbf{n}=0 \quad \text { on } \quad S^{+} \\
\widehat{\mu} \nabla \phi \cdot \mathbf{n}=0 \quad \text { on } \quad S_{0}
\end{gathered}
$$




$$
\begin{gathered}
\mu_{z} \frac{\partial \phi}{\partial z}-U_{n} \phi=-\nu^{s} \phi \quad \text { on } \quad S_{H}^{-} \\
\mu_{z} \frac{\partial \phi}{\partial z}=-\nu^{s} \phi \quad \text { on } \quad S_{H}^{+} \\
\nabla \cdot \mathbf{U}=\frac{\partial u}{\partial x}+\frac{\partial v}{\partial y}+\frac{\partial w}{\partial z}=0 \text { in } \mathbf{D} .
\end{gathered}
$$

Here $\phi(\mathbf{r}, t) \geq 0$ represents the concentration of one primary pollutant with a distribution $\phi^{0}(\mathbf{r})$ in $\mathbf{D}$ at initial moment $t=0, \sigma(\mathbf{r}, t) \geq 0$ is the chemical transformation coefficient, and $\mu(\mathbf{r}, t)>0$ and $\widehat{\mu}(\mathbf{r}, t)>0$ are the turbulent diffusion arrays,

$$
\begin{gathered}
\mu=\left(\begin{array}{ll}
\mu_{x}(\mathbf{r}, t) & 0 \\
0 & \mu_{y}(\mathbf{r}, t)
\end{array}\right), \\
\widehat{\mu}=\left(\begin{array}{lll}
\mu_{x}(\mathbf{r}, t) & 0 & 0 \\
0 & \mu_{y}(\mathbf{r}, t) & 0 \\
0 & 0 & \mu_{z}(\mathbf{r}, t)
\end{array}\right),
\end{gathered}
$$

respectively.

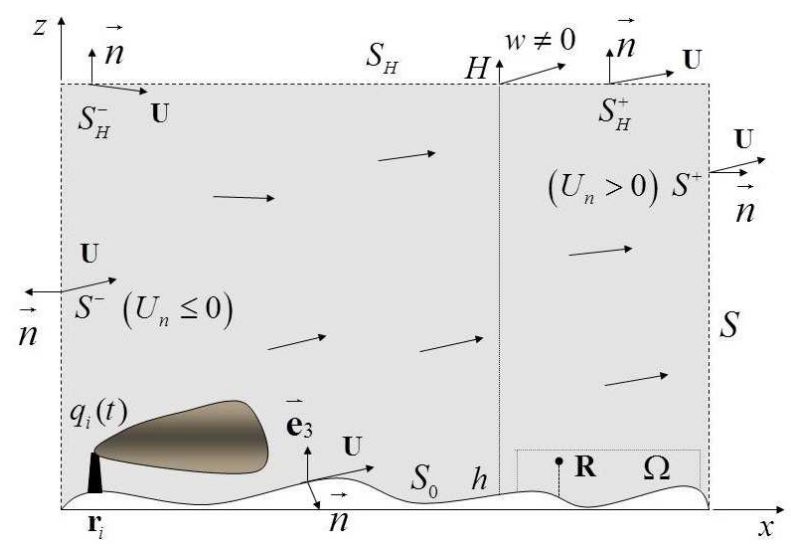

Figure 1: Cross section of region $\mathbf{D}$.

In equation (1), the term $\nabla \cdot \phi^{s}$ describes the change of concentration of particles in unit time because of sedimentation with constant velocity $\nu^{s}>0$. It is assumed that the wind velocity $\mathbf{U}(\mathbf{r}, t)=(u, v, w)$ is known in $\mathbf{D}$ and satisfies the continuity equation (9).

Assume that the forcing,

$$
f(\mathbf{r}, t)=\sum_{i=1}^{N} f_{i}(\mathbf{r}, t)
$$


is formed by point, line or area emission sources $f_{i}(\mathbf{r}, t)$ located in the domain $\mathbf{D}, i=1, \ldots, N$. Each point emission source $f_{j}(\mathbf{r}, t)$ can be described through its emission rate $Q_{j}(t)$ and emission site $\mathbf{r}_{j}$, that is, $f_{j}(\mathbf{r}, t)=Q_{j}(t) \delta\left(\mathbf{r}-\mathbf{r}_{j}\right)$, where $\delta\left(\mathbf{r}-\mathbf{r}_{j}\right)$ is the Dirac delta centered at $\mathbf{r}_{j} \in \mathbf{D}$. The domain of function $f_{k}(\mathbf{r}, t)$ is restricted to a line $\Gamma_{k} \subset D$ in the case of a line source, and to a two-dimensional set $A_{l} \subset D$ in the case of an area source $f_{l}(\mathbf{r}, t)$. It is important to note that each linearly distributed source, as well as each source distributed over an area, can be approximated by the sum of point sources [16]. However, the formulation of the control problem does not require such transformation, because such details are part of the numerical scheme used to solve the dispersion model (1)-(9).

The conditions on the open boundary $\partial \mathbf{D}$ of domain $\mathbf{D}$ lead to the wellposed problem in the sense of Hadamard [9]. We denote by $U_{n}=\mathbf{U} \cdot \mathbf{n}$ the projection of the velocity $\mathbf{U}$ on the outward unit normal $\mathbf{n}$ to the boundary $S$, which is divided into the outflow part $S^{+}$where $U_{n} \geq 0$ (advective pollution flow is directed out of $\mathbf{D}$ ) and the inflow part $S^{-}$where $U_{n}<0$ (advective pollution flow is directed into $\mathbf{D}$ ). The region $\mathbf{D}$ is assumed to be large enough to include all important pollution sources. Thus, we suppose that there is no sources outside $\mathbf{D}$, and by condition (4), the combined (diffusive plus advective) pollution flow is zero on the inflow part $S^{-}$. The pollution flow is non-zero only on $S^{+}$, besides, according to (5), the diffusive pollution flow on $S^{+}$is assumed to be negligible as compared with the corresponding advective pollution flow. The conditions (7) and (8) have similar meanings on $S_{H}$, where the sedimentation of the particles has been taken into account. Equation (6) indicates no flow of the substances through $S_{0}$, since $\mathbf{U} \cdot \mathbf{n}$ and $\nu^{s}$ are both zero on the irregular terrain (see Fig. 1). In general, equations (7) and (8) are necessary because $w=0$ on $S_{0}$ and (9) lead to a non-zero vertical velocity component at $S_{H}$ :

$$
w(x, y, z, t)=-\int_{0}^{z}\left(\frac{\partial u}{\partial x}+\frac{\partial v}{\partial y}\right) d z .
$$

The boundary conditions are mathematically good, because problem (1)-(9) is well posed, that is, its solution exists, is unique and continuously depends on the initial condition and forcing [21]. This follows from the fact that the problem operator

$$
A \phi=\mathbf{U} \cdot \nabla \phi+\sigma \phi-\nabla \cdot(\mu \nabla \phi)-\frac{\partial}{\partial z} \mu_{z} \frac{\partial \phi}{\partial z}+\nabla \cdot \phi^{s}
$$

is linear and nonnegative:

$$
(A \phi, \phi)=\int_{D}\left\|\widehat{\mu}^{\frac{1}{2}} \nabla \phi\right\|_{2}^{2} d \mathbf{r}+\int_{D} \sigma \phi^{2} d \mathbf{r}+\frac{1}{2} \int_{S_{0}} \nu^{s} \phi^{2}\left|\mathbf{e}_{3} \cdot \mathbf{n}\right| d S
$$




$$
+\int_{S_{H}} \nu^{s} \phi^{2} d S+\frac{1}{2}\left\{\int_{S^{+} \cup S_{H}^{+}} U_{n} \phi^{2} d S-\int_{S^{-} \cup S_{H}^{-}} U_{n} \phi^{2} d S\right\} \geq 0 .
$$

Here $(\phi, \eta)=\int_{\mathbf{D}} \phi \eta d \mathbf{r}$ and $\|\phi\|_{2}=\left(\int_{\mathbf{D}} \phi^{2} d \mathbf{r}\right)^{1 / 2}$ define the inner product and the norm in the Hilbert space $L_{2}(D)$, respectively. It can be shown [21] that

$$
\|\phi\|_{2} \leq T \max _{0 \leq t \leq T}\|f(\mathbf{r}, t)\|_{2}+\left\|\phi^{0}\right\|_{2} .
$$

The boundary conditions are also physically appropriate, since the integration of transport equation (1) over domain $\mathbf{D}$ leads to a mass balance equation

$$
\begin{aligned}
\frac{\partial}{\partial t} \int_{\mathbf{D}} \phi d \mathbf{r}=\sum_{i=1}^{N} \int_{\mathbf{D}} f_{i}(\mathbf{r}, t) d \mathbf{r}-\int_{S^{+} \cup S_{H}^{+}} U_{n} \phi d S \\
-\int_{\mathbf{D}} \sigma \phi d \mathbf{r}-\int_{S_{0}} \nu^{s} \phi\left|\mathbf{e}_{3} \cdot \mathbf{n}\right| d S .
\end{aligned}
$$

Thus, the total mass of the pollutants increases due to the nonzero emission rates $f_{i}(\mathbf{r}, t)$, and decreases because of advective outflow across $S^{+} \cup S_{H}^{+}$, chemical transformations $(\sigma \neq 0)$ and settlement on the ground $\left(\nu^{s} \neq 0\right)$.

Finally, we point out that the numerical solution of the dispersion model (1)-(9) is obtained with a balanced and absolutely stable second-order finitedifference scheme based on the application of the splitting method and CrankNicolson scheme [3], [12], [20].

\section{Optimal Control}

Let the meteorological conditions and air quality be predicted during a time interval $(0, T)$ by the dispersion model (1)-(9) in combination with a weather forecast model. Suppose that the air quality forecast obtained with emission rates $f_{i}(\mathbf{r}, t)(i=1, \ldots, N)$ is unfavorable, that is $\phi(\mathbf{r}, t)>J_{0}$ at some points of a zone $\Omega \subset \mathbf{D}$ and some moments of the time interval $(0, T)$, where $J_{0}$ is the air quality norm for the pollutant. Then, in order to prevent an excessive concentration of the pollutant in the zone $\Omega$, a short-term control must be applied to establish a suitable intensity of all pollution sources within $(0, T)$. In other words, we should determine in the time interval $(0, T)$ reduced emission rates, optimal in the sense that the air quality standard will be satisfied:

$$
\phi(\mathbf{r}, t) \leq J_{0}, \text { for any } \mathbf{r} \in \Omega \text { and } t \in(0, T)
$$


In order to simplify the discussion we write the dispersion model (1)-(9) in the form

$$
\frac{\partial \phi}{\partial t}+A \phi=\sum_{i=1}^{N} f_{i}(\mathbf{r}, t), \quad \phi(\mathbf{r}, 0)=\phi^{0}(\mathbf{r}) \quad \text { in } \mathbf{D} .
$$

The boundary conditions (4) to (8) and equation (9) are omitted because they do not depend on the forcing and initial condition. At the same time, we assume that the solutions of dispersion model (1)-(9) for different forcing and initial conditions are continuos functions. Indeed, the Laplace operator which is included in the definition of the operator $A$ guarantees not only the continuity of the solution, but also the continuity of its derivatives.

The control strategy for the emission rates of sources of pollutant consists of finding nonnegative damping coefficients $\lambda_{i} \leq 1$ which determine the new emission rates as

$$
\lambda_{i} f_{i}(\mathbf{r}, t), \quad i=1, \ldots, N,
$$

where $\left(1-\lambda_{i}\right) \times 100$ represents the percentage short-term decrease of emissions of the $i$ th pollution source. The aim of parameters $\lambda_{i}$ is the fulfillment of the environmental condition (17) for the solution $\varphi$ of the following dispersion problem:

$$
\frac{\partial \varphi}{\partial t}+A \varphi=\sum_{i=1}^{N} \lambda_{i} f_{i}(\mathbf{r}, t), \quad \varphi(\mathbf{r}, 0)=\phi^{0}(\mathbf{r}) \quad \text { in } \quad \mathbf{D} .
$$

Since this objective can be accomplished by different sets of such parameters, the optimal values will be obtained by means of a suitable optimization process.

We now introduce concentration functions $C_{i}=C_{i}(\mathbf{r}, t), i=0, \ldots, N$, as the solutions of the following dispersion problems:

$$
\frac{\partial C_{0}}{\partial t}+A C_{0}=0, \quad C_{0}(\mathbf{r}, 0)=\phi^{0}(\mathbf{r}) \quad \text { in } \quad \mathbf{D},
$$

and

$$
\frac{\partial C_{i}}{\partial t}+A C_{i}=f_{i}(\mathbf{r}, t), \quad C_{i}(\mathbf{r}, 0)=0 \quad \text { in } \quad \mathbf{D}, \quad i=1, \ldots, N .
$$

Note that when $C_{j}(\mathbf{r}, t)=0$, for any $\mathbf{r} \in \Omega$ and any instant $t \in(0, T)$, then the $j$ th source can be excluded from the control problem. Hereafter we consider that such sources have been identified and removed, i.e., we assume that all $N$ sources of the model (1)-(9) are responsible for polluting the zone $\Omega$.

Since the dispersion model (1)-(9) is linear and has unique solution, one can write the solution of problem (20) as a linear combination of the concentration 
functions $C_{i}$,

$$
\varphi(\mathbf{r}, t)=C_{0}(\mathbf{r}, t)+\sum_{i=1}^{N} \lambda_{i} C_{i}(\mathbf{r}, t), \quad \mathbf{r} \in \mathbf{D} \quad \text { and } \quad t \in(0, T)
$$

for any set of nonnegative damping coefficients $\lambda_{i} \leq 1, i=1, \ldots, N$.

Due to (23), the environmental condition (17) is equivalent to the following relation:

$$
\sum_{i=1}^{N} \lambda_{i} C_{i}(\mathbf{r}, t) \leq J_{0}-C_{0}(\mathbf{r}, t), \quad \mathbf{r} \in \Omega \quad \text { and } \quad t \in(0, T) .
$$

It should be noted that the function

$$
\alpha(\mathbf{r}, t)=J_{0}-C_{0}(\mathbf{r}, t), \quad \mathbf{r} \in \Omega \quad \text { and } \quad t \in(0, T)
$$

defined by the right-hand side of inequality (24), indicates different cases of the control problem.

\section{Control cases:}

1. If $\alpha \leq 0$ at a point $\mathbf{r} \in \Omega$ and $t \in(0, T)$, then the damping coefficients must be taken as $\lambda_{i}=0$ for any $i$, namely, all emissions must be stopped in order to prevent dangerous concentrations of the pollutant. This case appears when the initial pollutant concentration $\phi^{0}(\mathbf{r})$ is sufficiently high.

2. If $\alpha(\mathbf{r}, t)>0$ for all $\mathbf{r} \in \Omega$ and $t \in(0, T)$, then a quadratic programming problem is posed to calculate optimal values of the damping coefficients $\lambda_{i}$. In order to formulate such optimization problem we establish the following theorem.

Theorem 1. Let $M_{i}=\max \left\{C_{i}(\mathbf{r}, t),(\mathbf{r}, t) \in \overline{\Omega \times(0, T)}\right\}>0, i=1, \ldots, N$, and $\alpha_{0}=\min \{\alpha(\mathbf{r}, t),(\mathbf{r}, t) \in \overline{\Omega \times(0, T)}\} \geq 0$. Then the environmental condition (17) is fulfilled for any set of nonnegative damping coefficients $\lambda_{i} \leq 1$ $(i=1, \ldots, N)$ such that

$$
\sum_{i=1}^{N} \lambda_{i} M_{i} \leq \alpha_{0}
$$

Proof. The constants $M_{i}$ and $\alpha_{0}$ are well defined by means of the Weierstrass theorem for continuous functions optimized on compact sets of an Euclidean space [11]. Now, using the extreme values as upper and lower bounds, 
and inequality (26), we can establish that

$$
\begin{gathered}
\sum_{i=1}^{N} \lambda_{i} C_{i}(\mathbf{r}, t) \leq \sum_{i=1}^{N} \lambda_{i} M_{i} \leq \alpha_{0} \\
\leq \alpha(\mathbf{r}, t)=J_{0}-C_{0}(\mathbf{r}, t), \quad \mathbf{r} \in \Omega \quad \text { and } \quad t \in(0, T) .
\end{gathered}
$$

From equations (27) and (23) we obtain that

$$
\varphi(\mathbf{r}, t)=C_{0}(\mathbf{r}, t)+\sum_{i=1}^{N} \lambda_{i} C_{i}(\mathbf{r}, t) \leq J_{0}, \quad \mathbf{r} \in \Omega \quad \text { and } \quad t \in(0, T),
$$

and consequently, condition (17) is satisfied.

With the aim to choose an optimal set of nonnegative damping coefficients which satisfies the relation (26) for $\alpha_{0}>0$, let us define the relative cost function as follows:

$$
\begin{gathered}
F\left(\lambda_{1}, \ldots, \lambda_{N}\right)=\sum_{i=1}^{N} c_{i}^{2} \frac{\int_{0}^{T} \int_{\mathbf{D}}\left[f_{i}(\mathbf{r}, t)-\lambda_{i} f_{i}(\mathbf{r}, t)\right]^{2} d \mathbf{r} d t}{\int_{0}^{T} \int_{\mathbf{D}}\left[f_{i}(\mathbf{r}, t)\right]^{2} d \mathbf{r} d t} \\
=\sum_{i=1}^{N} c_{i}^{2}\left(1-\lambda_{i}\right)^{2} .
\end{gathered}
$$

Here each coefficient $c_{i}>0, i=1, \ldots, N$, represents the price to be paid by the $i$ th-source according to the control strategy, that is, for reducing its emissions by $\left(1-\lambda_{i}\right) \times 100$ percentage. Taking into account the cost function (28) and condition (26), the optimal control problem is to determine the damping coefficients $\lambda_{i}$ by solving the following quadratic programming problem:

$$
\begin{array}{ll}
\text { Minimize } & F\left(\lambda_{1}, \ldots, \lambda_{N}\right)=\sum_{i=1}^{N} c_{i}^{2}\left(1-\lambda_{i}\right)^{2}, \\
\text { subject to: } & \left\{\begin{array}{c}
\sum_{i=1}^{N} \lambda_{i} M_{i} \leq \alpha_{0}, \quad \text { and } \\
0 \leq \lambda_{i} \leq 1, i=1, \ldots, N
\end{array}\right\} .
\end{array}
$$

Note that due to Theorem 1, any set of the damping coefficients from the feasible space (30) also fulfills the environmental condition (17). Besides, the quadratic programming problem (29)-(30) can be written as follows:

$$
\text { Minimize } F\left(x_{1}, \ldots, x_{N}\right)=\sum_{i=1}^{N}\left(x_{i}-x_{i}^{0}\right)^{2},
$$




$$
\text { subject to: }\left\{\begin{array}{c}
\sum_{i=1}^{N} b_{i} x_{i} \leq \alpha_{0}, \quad \text { and } \\
0 \leq x_{i} \leq x_{i}^{0}, i=1, \ldots, N
\end{array}\right\} .
$$

where $x_{i}=c_{i} \lambda_{i}, x_{i}^{0}=c_{i}$ and $b_{i}=M_{i} / c_{i}$. Observe that the optimal control of emissions has been reduced to an optimization problem in $\mathbf{R}^{N}$ whose feasible space $\mathcal{F}$ is defined by the constraints (32). Also note that the point $\left\{x_{i}^{0}\right\}$ is not in the feasible space $\mathcal{F}$ because $\sum_{i=1}^{N} b_{i} x_{i}^{0}>\alpha_{0}$, otherwise, there is not a problem of excessive pollution in $\Omega$,

$$
\sum_{i=1}^{N} C_{i}(\mathbf{r}, t) \leq \sum_{i=1}^{N} M_{i}=\sum_{i=1}^{N} b_{i} x_{i}^{0} \leq \alpha_{0} \leq \alpha(\mathbf{r}, t)=J_{0}-C_{0}(\mathbf{r}, t) .
$$

Theorem 2. The solution of the quadratic programming problem (31)(32) is unique

Proof. The feasible space $\mathcal{F}$ is a nonempty set since $\mathbf{0}=\{0\} \in \mathcal{F}$, moreover, it is a bounded set since $\left\{x_{i}\right\} \in \mathcal{F}$ implies that $\left\|\left\{x_{i}\right\}\right\| \leq\left\|\left\{x_{i}^{0}\right\}\right\|=$ constant, for the Euclidean norm in $\mathbf{R}^{N}$. In order to prove that $\mathcal{F}$ is a closed set we define the function $g$ as follows, $g\left(x_{1}, \ldots, x_{N}\right)=\sum_{i=1}^{N} b_{i} x_{i} . g$ is a continuous function in $\mathbf{R}^{N}$ and $\left(-\infty, \alpha_{0}\right]$ is a closed interval in $\mathbf{R}^{1}$ then $g^{-1}\left(-\infty, \alpha_{0}\right]$ is a closed set in $\mathbf{R}^{N}[6]$. By the same argument the sets $P_{i}^{-1}\left[0, x_{i}^{0}\right]$ are closed, where $P_{i}$ is the projection function from $\mathbf{R}^{N}$ to $\mathbf{R}^{1}$ for the $i$ th-component of vector $\left\{x_{i}\right\}$ and $\left[0, x_{i}^{0}\right]$ is a closed interval in $\mathbf{R}^{1}$. Finally, the feasible set $\mathcal{F}$ is an intersection of closed sets in $\mathbf{R}^{N}, \mathcal{F}=g^{-1}\left(-\infty, \alpha_{0}\right] \cap P_{1}^{-1}\left[0, x_{1}^{0}\right] \cap \cdots \cap P_{N}^{-1}\left[0, x_{N}^{0}\right]$, therefore, $\mathcal{F}$ is a closed set in $\mathbf{R}^{N}$ [6]. With these properties the feasible space $\mathcal{F}$ is a nonempty compact set in $\mathbf{R}^{N}$. By using the Weierstrass Theorem [11] we conclude that the solution of the quadratic programming problem (31)-(32) always exists because of the feasibility space $\mathcal{F}$ is a nonempty compact set in $\mathbf{R}^{N}$ and the objective function $F$ is continuous.

On the other hand, the feasible space $\mathcal{F}$ is a convex set in $\mathbf{R}^{N}$ because it is the intersection of convex sets in $\mathbf{R}^{N}$, and $F$ is a strictly convex function because its Hessian $\left(\partial^{2} F / \partial x_{i} \partial x_{j}\right)_{N \times N}=2 I$ is a positive definite matrix [11], such characteristics determine that the solution of the quadratic programming problem (31)-(32), which is a global minimum, is unique [11].

The solution of problem (31)-(32) is the point $\left\{x_{i}^{*}\right\}$ in $\mathcal{F}$ that minimizes the distance from the feasible space $\mathcal{F}$ to the point $\left\{x_{i}^{0}\right\}$. It is clear that in the 
Euclidean space $\mathbf{R}^{N}$ such solution is the orthogonal projection of point $\left\{x_{i}^{0}\right\}$ on the set defined by the equation $\sum_{i=1}^{N} b_{i} x_{i}=\alpha_{0}$ (see Fig. 2), that is, the solution of control problem always satisfies the constraint $\sum_{i=1}^{N} b_{i} x_{i}=\alpha_{0}$. In this way, the problem (31)-(32) can be replaced by a simpler optimization problem whose solution is also the point $\left\{x_{i}^{*}\right\}$ :

$$
\begin{aligned}
& \text { Minimize } \quad F\left(x_{1}, \ldots, x_{N}\right)=\sum_{i=1}^{N}\left(x_{i}-x_{i}^{0}\right)^{2}, \\
& \text { subject to: }\left\{\begin{array}{l}
\sum_{i=1}^{N} b_{i} x_{i}=\alpha_{0}, \quad \text { and } \\
0 \leq x_{i}, i=1, \ldots, N
\end{array}\right\} .
\end{aligned}
$$

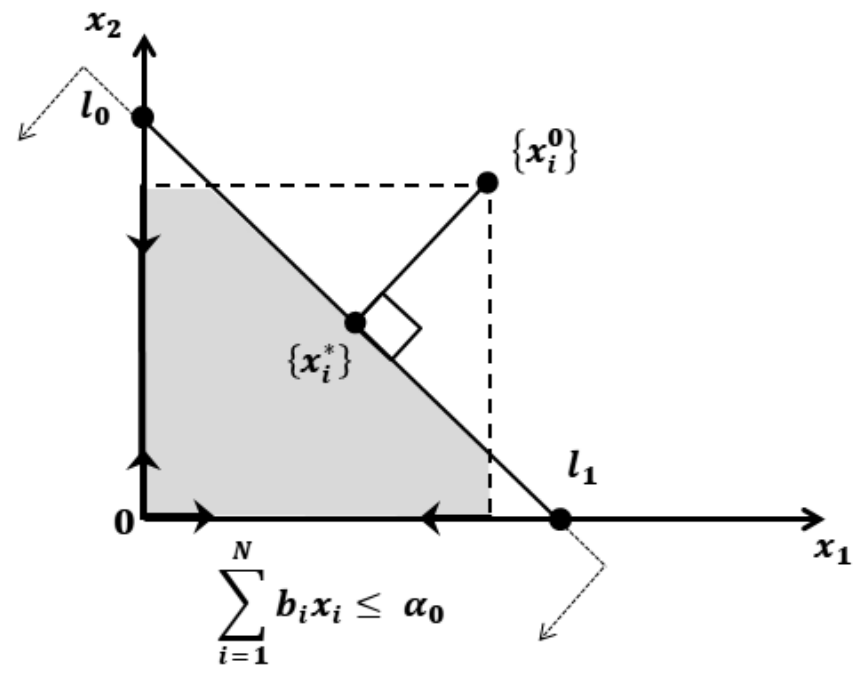

Figure 2: The optimal solution $\left\{x_{i}^{*}\right\}$ as an orthogonal projection of point $\left\{x_{i}^{0}\right\}$ on the feasible set $\mathcal{F}$ (gray zone).

We point out that the problem (33)-(34) is efficiently solved by means of the algorithm of successive orthogonal projections [15].

Algorithm of successive orthogonal projections. The optimization problem solution $\mathbf{x}^{*}=\left\{x_{j}^{*}\right\}$ is found by using the differential characterization 
theorem [11] which states that $\mathbf{x}^{*}$ is the solution if $-\nabla F\left(\mathbf{x}^{*}\right) \cdot\left(\mathbf{x}-\mathbf{x}^{*}\right) \leq 0$, for all $\mathbf{x} \in \mathcal{F}\left(\mathbf{x} \neq \mathbf{x}^{*}\right)$.

The first approximation of $\mathrm{x}^{*}$ is obtained as an orthogonal projection, or equivalently, as the minimization process using the method of Lagrange multipliers [11]:

$$
\begin{gathered}
\beta=2\left(\sum_{i=1}^{N} b_{i} x_{i}^{0}-\alpha_{0}\right)\left(\sum_{i=1}^{N} b_{i}^{2}\right)^{-1}, \\
x_{j}^{*}=x_{j}^{0}-0.5 \beta b_{j}, \quad j=1, \ldots, N .
\end{gathered}
$$

This approximation satisfies the condition $\sum_{j=1}^{N} b_{j} x_{j}^{*}=\alpha_{0}$. To improve the approximation, successive orthogonal projections are used.

Step I. If $x_{j}^{*} \geq 0$ for $j=1, \ldots, N$, then $\mathbf{x}^{*} \in \mathcal{F}$ and

$$
-\nabla F\left(\mathbf{x}^{*}\right) \cdot\left(\mathbf{x}-\mathbf{x}^{*}\right)=\beta \sum_{j=1}^{N} b_{j} x_{j}-\beta \sum_{j=1}^{N} b_{j} x_{j}^{*}=\beta \alpha_{0}-\beta \alpha_{0}=0,
$$

for all $\mathbf{x}^{*} \in \mathcal{F}\left(\mathbf{x} \neq \mathbf{x}^{*}\right)$. By the differential characterization theorem, $\mathbf{x}^{*}$ is the minimum.

Step II. If at least one component of $\mathrm{x}^{*}$ is negative then the projection needs to be redefined. Without loss of generality, assume that $x_{j}^{*} \leq 0$ for $j=l+1, \ldots, N$ (at any rate one of the components must be always positive, since $\left.\alpha_{0}>0\right)$. Let us define $x_{j}^{* *}=0$ for $j=l+1, \ldots, N$, and the rest variables are taken from the new projection with restricted $F$ and feasible space $\mathcal{F}$ :

$$
x_{j}^{* *}=x_{j}^{0}-0.5 \eta b_{j}, \quad j=1, \ldots l, \quad \eta=2 \frac{\sum_{i=1}^{l} b_{i} x_{i}^{0}-\alpha_{0}}{\sum_{i=1}^{l} b_{i}^{2}} .
$$

This approximation also leads to the condition $\sum_{j=1}^{N} b_{j} x_{j}^{* *}=\alpha_{0}$. If $x_{j}^{* *} \geq 0$ for $j=1, \ldots, l$, then $\mathbf{x}^{* *} \in \mathcal{F}$ and

$$
-\nabla F\left(\mathbf{x}^{* *}\right) \cdot\left(\mathbf{x}-\mathbf{x}^{* *}\right)=2 \sum_{i=l+1}^{N} x_{i}\left(x_{i}^{0}-\frac{\eta}{2} b_{i}\right), \quad \mathbf{x} \in \mathcal{F}, \mathbf{x} \neq \mathbf{x}^{*} .
$$

Since $\sum_{i=1}^{l} b_{i} x_{i}^{*}>\alpha_{0}$, it follows from (35) that $\eta>\beta$. This inequality, together with $(35)$, leads to $-\nabla F\left(\mathbf{x}^{* *}\right) \cdot\left(\mathbf{x}-\mathbf{x}^{* *}\right) \leq 2 \sum_{i=l+1}^{N} x_{i} x_{i}^{*} \leq 0$ for all $\mathbf{x} \in \mathcal{F}\left(\mathbf{x} \neq \mathbf{x}^{*}\right)$. Due to the differential characterization theorem, $\mathbf{x}^{* *}$ is the minimum.

This algorithm converges to the exact solution at most in $N$ iterations (projections), because $\alpha_{0}>0$. Moreover, it is not computer time consuming, since 
the number of arithmetic operations does not exceed $3\left(N^{2}+N\right)$. For example, if a computer realizes $1,000,000 \mathrm{op} / \mathrm{s}$ then all the optimal variables $x_{i}^{*}$ can be determined at most in $3.5 \mathrm{~s}$ for $N=1000$, and in $75.5 \mathrm{~s}$ for $N=5000$. Thus, every time when a short-term forecast is unfavorable, the quadratic programming problem (29)-(30) can rapidly be solved. This property is especially important when the number of emission sources is large.

We point out that this control method could result rigorous for some pollution sources due to the fact that it is based on condition (26), according to which the emissions are limited through the maximum values $M_{i}$. However, this method is the only option during some pollution events. For example, when each pollution source generates a maximum value of concentration $M_{i}$ around the same point in $\Omega$ then the maximum value of the pollutant concentration in the $\Omega$ zone is the sum $\sum_{i=1}^{N} M_{i}$, and hence, to satisfy the environmental condition (17) any control strategy must use the condition (26). To imagine this pollution event one can consider many pollution sources located on a line coinciding with the wind direction, at some point downwind on this line the concentration of the pollutant is the sum of the maximum contributions of all sources.

In order to estimate how large can be the damping coefficients for any control strategy, including the control method described in this section, we establish the following theorem.

Theorem 3. The upper bounds for the nonnegative damping coefficients in any control strategy that fulfills the environmental condition (17) are given as

$$
\lambda_{i}^{u}=\min \left\{1, J_{0} / M_{i}\right\}, i=1, \ldots, N .
$$

Proof. The environmental condition (17) means that

$$
C_{0}(\mathbf{r}, t)+\sum_{j=1}^{N} \lambda_{j} C_{j}(\mathbf{r}, t) \leq J_{0}, \quad \mathbf{r} \in \Omega \quad \text { and } \quad t \in(0, T) .
$$

Since $\lambda_{j} C_{j}(\mathbf{r}, t) \geq 0$ for all $j$, then for a fixed $i$

$$
\lambda_{i} C_{i}(\mathbf{r}, t) \leq C_{0}(\mathbf{r}, t)+\sum_{j=1}^{N} \lambda_{j} C_{j}(\mathbf{r}, t) \leq J_{0}, \quad \mathbf{r} \in \Omega \quad \text { and } \quad t \in(0, T),
$$

and hence, $\lambda_{i} C_{i}(\mathbf{r}, t) \leq J_{0}, \mathbf{r} \in \Omega$ and $t \in(0, T)$. In particular, such inequality holds for the maximum value, that is, $\lambda_{i} M_{i} \leq J_{0}$. Then (37) follows from this condition and the fact that $\lambda_{i} \leq 1$. 
Due to (37), to control the emissions for large values of $M_{i}$, one must pose small damping coefficients. Finally, it is important to observe that the upper bounds (37) are only the necessary conditions for any control strategy, but generally they are not sufficient conditions.

\section{An Example of Optimal Control}

In this section we consider a two-dimensional version of dispersion model (1)(9), [17] to estimate and control the concentration of one passive pollutant $\left(\mathrm{SO}_{2}\right)$. The method for the control of emission rates studied in the previous section is applied to a synthetic situation that involves two point sources. The aim is to reduce the concentration of sulphur dioxide below the sanitary norm.

Let us consider in the square domain $\mathbf{D}=(0,3) \times(0,3)$ of $9 \mathrm{~km}^{2}$ the two industrial (point) sources with coordinates $\mathbf{r}_{1}=(1.55,0.25)$ and $\mathbf{r}_{2}=(0.55,2.05)$, which emit sulphur dioxide with the following nonstationary rates (in $\mathrm{kg} / \mathrm{h}$ ):

$$
\begin{gathered}
Q_{1}(t)=\left\{\begin{array}{cc}
10000 t, & t \in[0,0.5) \\
5000, & t \in[0.5,7] \\
5000(8-t), & t \in(7,8.0]
\end{array},\right. \\
Q_{2}(t)=\left\{\begin{array}{cc}
5000 t, & t \in[0,0.5] \\
2500, & t \in(0.5,1] \\
2500+2000(t-1), & t \in(1,1.5) \\
3500, & t \in[1.5,7.5] \\
3500-7000(t-7.5), & t \in(7.5,8.0)
\end{array} .\right.
\end{gathered}
$$

Assume that the initial distribution of sulphur dioxide in $\mathbf{D}$ is $\phi(\mathbf{r}, 0)=0$, while the parameters $\sigma=0.36 \mathrm{~h}^{-1}$ and $\mu=1.8 \mathrm{~km}^{2} \mathrm{~h}^{-1}$ for the dispersion model are taken from Shir and Shieh [19]. The wind velocity $\mathbf{U}=(u, v)$ is generated by the stream function $\psi(\mathbf{r})=(5 / 3) x^{2}-5 y$, and hence, the velocity components $u=-\partial \psi / \partial y=-5$ and $v=\partial \psi / \partial x$ satisfy the continuity equation (9): $\nabla \cdot \mathbf{U}=0$. We consider the control problem for the concentration of sulphur dioxide in the zone $\Omega=[1,2] \times[1,2]$ during the whole time interval $(0, T)$, where $T=8 \mathrm{~h}$. In order to observe the evolution of concentration with time in $\Omega$, we consider five monitoring points distributed in this area: $R_{1}=(1.05,1.05), R_{2}=(1.95,1.05), R_{3}=(1.55,1.55), R_{4}=(1.05,1.95)$ and $R_{5}=(1.95,1.95)$. Figure 3 shows the respective concentrations $\phi\left(R_{i}, t\right), i=$ $1, . ., 5$, and the allowed limit for the pollutant concentration $J_{0}=210 \mu \mathrm{gm}^{3}$ for the eight hours exposure [23]. We note that at points $R_{2}, R_{3}, R_{4}$ and $R_{5}$, the concentrations are above this limit for five hours, from $t=2$ to $t=7$, 
representing a violation of the corresponding sanitary norm. Thus, a control of emissions is necessary to protect the zone $\Omega$.

In order to apply the control method to this example we note that due to the initial condition $\phi(\mathbf{r}, 0)=0$ we obtain that $\alpha(\mathbf{r}, t)=J_{0}-C_{0}(\mathbf{r}, t)=J_{0}$, and hence, $\alpha_{0}=J_{0}$. Besides, the solutions of problems (22), calculated for each point source, allow to estimate the following maximum values of concentration: $M_{1}=363.5665$ and $M_{2}=317.1413$. Finally, we assume the same values of cost for each point source, namely, $c_{1}=c_{2}=1$.

By using Theorem 3 we estimate the following upper bounds for the damping coefficients: $\lambda_{1}^{u}=0.5776$ and $\lambda_{2}^{u}=0.6621$. Note that in this example, each pollution source must be severely restricted due to the fact that they are responsible for very high concentrations of the pollutant.

All this information allows us to pose and solve the quadratic programming problem (33)-(34). The algorithm of successive orthogonal projections converges in one iteration, that is, we obtain the solution of problem (33)-(34) only by the application of equation (35). The optimal damping coefficients are $\lambda_{1}^{*}=0.2648$ and $\lambda_{2}^{*}=0.3586$. Figure 4 shows the respective concentrations $\phi\left(R_{i}, t\right), i=1, . ., 5$, which were obtained for the new emission rates defined through equation (19). In all the monitoring sites $R_{i}, i=1, . ., 5$, the concentration of the pollutant satisfies the air quality norm in the whole time interval $(0, T), T=8 h$. Moreover, by Theorem 1 it is sure that the environmental condition $\phi(\mathbf{r}, t) \leq J_{0}$ is fulfilled for all $\mathbf{r} \in \Omega$ and $t \in(0, T)$.

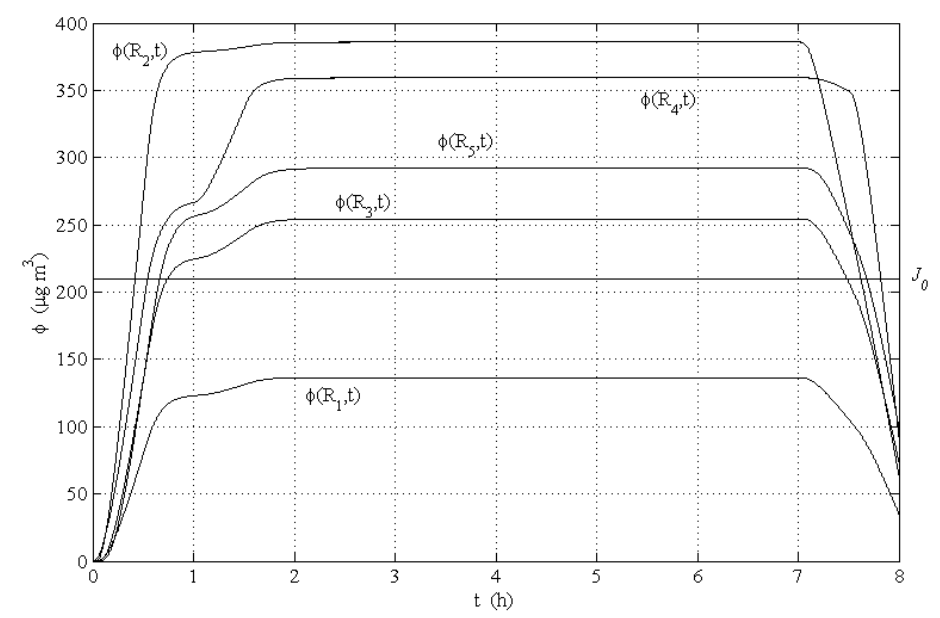

Figure 3: The evolution in time of the pollutant concentration at five monitoring sites in $\Omega$ before applying the control of emissions. 


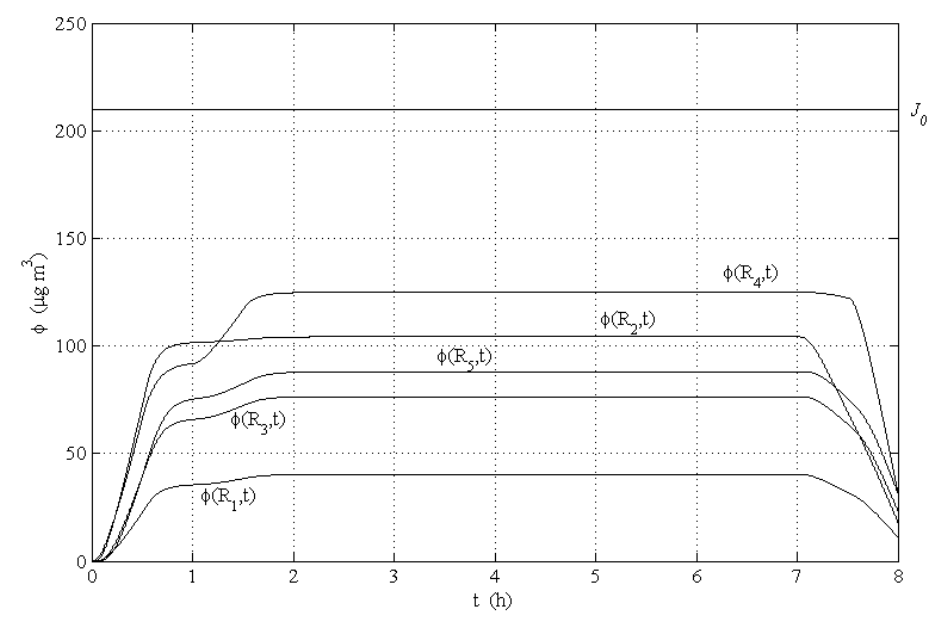

Figure 4: The evolution in time of the pollutant concentration at five monitoring sites in $\Omega$ after applying the control of emissions.

\section{Conclusions}

Progress in numerical short-term weather forecasting and modeling of pollution transport has opened up new opportunities for developing methods that not only can predict pollutant concentrations but also control emission rates to prevent dangerous levels of concentrations in the case of weak dispersion conditions in the atmosphere.

The development of various control strategies is based on using different optimization formulations which depend on the ecological goals, technological restrictions and the minimization of the cost that the sources of pollution must pay for reducing their emissions. In this work, we have posed and analyzed a quadratic programming problem with the aim to determine optimal emission rates of the pollution sources and meet the standards of air quality at every point in a zone and each instant in a time interval. The existence and uniqueness of a solution of the optimal control problem is proved. Also, an efficient algorithm of successive orthogonal projections is applied to calculate the optimal solution. Note two important advantages of the new control method. At first, it can be applied to any linear dispersion model. To this end, it is necessary to calculate various particular solutions of such model and the respective maximum values of 
these solutions. And secondly, the control method is useful to obtain damping coefficients for point, line and area sources of pollution.

\section{Acknowledgments}

This research was supported by Grants 25170 and 14539 of the National System of Investigators (SNI-CONACyT, México).

\section{References}

[1] S. Cheng, J. Li, B. Feng, Y. Jin, R. Hao, A gaussian-box modeling approach for urban air quality management in a Northern Chinese City - II. Pollutant emission abatement, Water, Air and Soil Pollution, 178, No 1-4 (2007), $15-36$.

[2] W.W. Cooper, H. Hemphill, Z. Huang, S. Li, V. Lelas, D.W. Sullivan, Survey of mathematical programming models in air pollution management, European Journal of Operational Research, 96, No 1 (1996), 1-35.

[3] J. Crank, P. Nicolson, A practical method for numerical evaluation of solutions of partial differential equations of the heat conduction type, Proc. Cambridge Philos. Soc., 43, No 1 (1947), 50-67.

[4] L. Feng, H. Fei, Z. Jiang, Adjoint method for the optimum planning of industrial pollutant sources, Science in China, Ser. D: Earth Sciences, 48, No 8 (2005), 1270-1279.

[5] R.C. Flagan, J.H. Seinfeld, Fundamentals of Air Pollution Engineering, Prentice-Hall (1988).

[6] R.R. Goldberg, Methods of Real Analysis, John Wiley \& Sons (1976).

[7] C.A. Gough, M.J. Chadwick, B. Biewald, J. Kylenstierna, P.D. Bailey, S. Cinderby, Developing optimal abatement strategies for the effects of sulphur and nitrogen deposition on European scale, Water, Air and Soil Pollution, 85, No 4 (1995), 2601-2606.

[8] H.J. Greenberg, Mathematical programming models for environmental quality control, Operations Research, 43, No 4 (1995), 578-623. 
[9] J. Hadamard, Lectures on Cauchy's Problem in Linear Partial Differential Equations, Dover Publications (1923).

[10] R.E. Kohn, A Linear Programming Model for Air Pollution Control, MIT Press, Cambridge, Mass. (1978).

[11] D.G. Luenberger, Linear and Nonlinear Programming, Addison-Wesley, Mass. (1984).

[12] G.I. Marchuk. Mathematical Models in Environmental Problems, Elsevier, New York (1986).

[13] D. Parra-Guevara, Yu.N. Skiba, Chapter 7: Quadratic Programming Formulation for Controlling the Emissions of Air Pollution Point Sources, 207-247. In: Horizons in Computer Science Research, Vol. 14, Editor: T.S. Clary, Nova Science Publishers, Inc., NY (2017).

[14] D. Parra-Guevara, Yu.N. Skiba, A. Pérez-Sesma, A linear programming model for controlling air pollution, International Journal of Applied Mathematics, 23, No 3 (2010), 549-569.

[15] D. Parra-Guevara, Yu.N. Skiba. On optimal solution of an inverse air pollution problem: Theory and numerical approach. Mathematical and Computer Modelling, 43, No 7-8 (2006), 766-778.

[16] D. Parra-Guevara, Yu.N. Skiba, Elements of the mathematical modelling in the control of pollutants emissions, Ecological Modelling, 167, No 3 (2003), 263-275.

[17] D. Parra-Guevara, Yu.N. Skiba, Industrial pollution transport. Part 2: Control of industrial emissions, Environmental Modeling and Assessment, 5, No 3 (2000), 177-184.

[18] V.V. Penenko, V.F. Raputa. Some models for optimizing the operation of the atmospheric-pollution sources, Sov. Meteorol. Hidrology, 2 (1983), 46-54.

[19] C.C. Shir, L.J. Shieh, A generalized urban air pollution model and its application to the study of $\mathrm{SO}_{2}$ distributions in the St. Louis Metropolitan Area, J. Appl. Meteorology, 13, No 2 (1974), 185-204.

[20] Yu.N. Skiba, D. Parra-Guevara, Applications of Adjoint Equations to Problems of Dispersion and Control of Pollutants, Nova Science Publishers, Inc., New York (2015). 
[21] Yu.N. Skiba, D. Parra-Guevara, Industrial pollution transport. Part I: Formulation of the problem and air pollution estimates, Env. Modeling and Assessment, 5, No 3 (2000), 169-175.

[22] A. Teller, The use of linear programming to estimate the cost of some alternative air pollution abatement policies, Art. No 20, In: Proc. of the IBM Scientific Computing Symposium on Water and Air Research Management, IBM, White Plains, NY (1968), 345-353.

[23] K. Wark, W.T. Davis, C.F. Warner. Air Pollution: Its Origin and Control, Addison-Wesley, Menlo Park, Calif., (1999). 
